\title{
Short and mid-term outcomes of multimodal treatment for locally-advanced non-small cell lung cancer in elderly patients
}

\author{
Stefano Bongiolatti ${ }^{1} \cdot$ Francesca Mazzoni $^{2} \cdot$ Alessandro Gonfiotti $^{1} \cdot$ Giulia Di Pierro $^{2} \cdot$ Alberto Salvicchi $^{1}$ (I) . \\ Sara Borgianni ${ }^{1} \cdot$ Katia Ferrari $^{3} \cdot$ Luca Voltolini $^{1}$
}

Received: 18 January 2020 / Accepted: 5 May 2020

(c) The Japanese Association for Thoracic Surgery 2020

\begin{abstract}
Objective Multimodality treatments are effective for locally advanced non-small cell lung cancer (LA-NSCLC) showing benefits in overall (OS) and disease-free survival (DFS), but these options are frequently denied to elderly patients.

Methods The objectives of this retrospective study were: to investigate mortality, morbidity and oncological outcomes of pulmonary resection after induction therapy (IT) for NSCLC in elderly patients. We divided the cohort into two: patients $<70$ years (group A) and patients $\geq 70$ years (group B). A multivariable logistic regression was built to identify factors associated with morbidity.

Results 77 patients underwent pulmonary resection after IT, 27 were aged $\geq 70$ years. Type of chemotherapy, surgical procedures, pathological stages were comparable between the two groups, while the preoperative use of chemo-radiation therapy regimen was more frequent in group A $(p=0.027)$. In-hospital mortality was similar, while the percentage of patients with complications ( $38 \%$ vs $48.1 \%, p=0.47)$ and the complication rate $(50 \%$ vs $77 \%, p=0.01)$ were higher in group $\mathrm{B}$, but the severity of complications was comparable. The multivariable analysis did not identify any risk factors associated with morbidity. OS at 3 years and DFS at 2 years were not different ( $61 \%$ vs $48.5 \%, p=0.64 ; 61.7 \%$ vs $44 \%, p=0.393$ ).

Conclusions Lung resection for LA-NSCLC after IT can be performed safely in selected elderly patients with favourable postoperative and mid-term oncological results.
\end{abstract}

Keywords NSCLC multimodal treatment $\cdot$ Neoadjuvant therapy $\cdot$ Elderly $\cdot$ Locally-advanced NSCLC $\cdot$ Survival

\section{Introduction}

The geriatric population is increasing worldwide and lung cancer is frequently diagnosed in elderly patients representing a leading cause of death. Age per se has been shown to be an important risk factor for morbidity and mortality after lung resection for non-small cell lung cancer (NSCLC) [1-6] and also the incidence of other diseases is more frequent in the older population then the association between these two conditions could preclude a complete oncological treatment.

Alberto Salvicchi

alberto.salvicchi@libero.it

1 Thoracic Surgery Unit, Careggi University Hospital, Largo Brambilla, 1, 50134 Florence, Italy

2 Medical Oncology Unit, Careggi University Hospital, Florence, Italy

3 Respiratory Medicine, Careggi University Hospital, Florence, Italy
Multimodality treatments have demonstrated clinical benefits in terms of overall and disease-free survival for locally advanced NSCLC (LA-NSCLC) [7-12]. Induction therapy (IT) has demonstrated some advantages: potential attack to micrometastases, improved systemic drug delivery and tolerability, better compliance with subsequent therapies, and also allows more time to identify unsuspected metastases or comorbidities contraindicating a definitive treatment as surgery [13]. The geriatric population is often underrepresented or excluded from clinical trials for the fear of higher toxicity and comorbidities and therefore there is a lack of specific data on therapeutic multimodal strategies in this population $[1,2,7]$. Some studies demonstrated higher toxicity and less benefits in the elderly subgroup [14], other confirmed the feasibility and efficacy of combined treatments [15].

The aim of our retrospective study was to analyze mortality and morbidity of lung resection after IT in patients 70 years or older identifying the presence of independent risk factors of postoperative complications. Furthermore, we 
examined also the oncological short and mid-term results to evaluate the feasibility and oncological adequacy of the multimodal treatment on the elderly.

\section{Materials and methods}

From June 2014 to December 2018, we retrospectively reviewed the medical records of all patients who received preoperative chemotherapy with or without radiation therapy and subsequently lung resection at Careggi University Hospital, Florence. Our institutional review board granted approval and waived the requirement for specific informed consent for this retrospective study.

Baseline and postoperative variables included demographics, comorbidities, pulmonary cardiovascular and renal assessment, preoperative clinical stage, pathological stage, hospital length of stay, duration of chest tubes, complications, and peri-operative mortality (between 30 days).

Clinical stage was assessed with whole-body computer tomographic scan (wb-CT), positron emission tomographic scan (PET-CT-scan), bronchoscopy, endobronchial or esophageal ultrasound (EBUS/EUS) and video-mediastinoscopy. Clinical and pathological stages were resumed with the American Joint Committee on Cancer $8^{\text {th }}$ Edition TNM Classification [16]. Stages obtained before the publication of the 8th Edition were re-checked and reported by the first author (B.S.).

All patients with LA-NSCLC were evaluated by the institutional Multidisciplinary Tumour Board (MTB) composed by expert oncologists, pulmonologists, radio-therapists, thoracic surgeons, and dedicated thoracic radiologists. At the weekly meeting, the individual treatment was decided based on the clinical stage and the most recent international guidelines $[17,18]$. In the absence of progression of disease at the re-staging assessment, including CT-scan and PET-CT-scan, patients were referred to thoracic surgery and dedicated anaesthesiology service to evaluate the operability.

During the study period, we compared all patients who underwent IT followed by radical surgery for LA-NSCLC dividing the population based on the age at the beginning of the treatment: the Group A consisted of patients aged $<70$ years while in the Group B were included patient aged $\geq 70$ years. The assignment of 70 years as a threshold to define elderly patients was arbitrary but is frequently used in the literature $[19,20]$. Follow-up was assessed by outpatient visits including interval medical history, physical examination, and enhanced contrast wb-CT scan every 6 months.

\section{Statistical analysis}

Statistical analysis was performed using SPSS 24.0 software (SPSS Inc., Chicago, IL). Continuous variables are expressed as median, mean values $\pm \mathrm{SD}$ and compared with unpaired $t$-test or non-parametric Mann-Whitney test, if not normally distributed. Categorical variables were analyzed using the $\chi^{2}$ test. Univariate and a multivariable logistic regression analysis were built to identify preoperative factors associated with overall morbidity, including a set of variables chosen on clinical relevance and entered in the multivariable model if $p<0.20$ (age, sex, Eastern Cooperative Oncology Group Performance Status (ECOG PS) [21], American Society of Anesthesiologists (ASA) score, Charlson Comorbidity Index (CCI) [22], number of comorbidities, pneumonectomy, chest wall resection, VATS approach, FEV1\% < 70).

The Kaplan-Meier method was used to calculate overall and disease-free survival. Overall survival (OS) was calculated from the date of the beginning of treatments to death or date of the last follow-up (31th December 2018); diseasefree survival (DFS) was calculated for those patients who received a radical resection from the date of operation to the date of the first evidence of recurrence. Differences in overall and disease-free survival between age classes were determined by log-rank analysis. Statistical significance for all tests was set at a probability value of less than 0.05 .

\section{Results}

During the study period, we identified 77 patients who underwent IT and consequently lung resection with systematic lymph node dissection.

Table 1 shows the preoperative characteristics, comorbidities, clinical-stage, type of induction treatment, therapeutic regimens, clinical response, surgical procedure, and pathological stage of the two groups. ECOG PS was worse for the elderly patient $(p=<0.01)$. Although the number and type of comorbidities were not different between the two groups, the CCI was statistically higher for patients of Group B $(p=0.03)$. Adenocarcinoma was more frequent in group A (62\% vs $40.7 \%)$, but this difference was not significant. The majority of patients were classified as clinical stage IIIA in both groups. The use of cisplatin-based chemotherapy regimen (CDDP) was more frequent in group A without statistical significance ( $80 \%$ vs $66.7 \%, p=0.24)$. However, in Group A, a large portion of patients underwent to combined preoperative chemo-radiation therapy regimen $(p=0.027)$. Regarding the surgical procedure, we had similar percentages of lobectomy, sleeve lobectomy, and pneumonectomy, but we noted a higher number of patients of Group A who underwent chest wall resection ( $20 \%$ vs $11.1 \%, p=0.52$ ).

The pathological stage did not differ between the two groups and we had a total of $10(12.9 \%)$ complete responses after IT. 
Table 1 Demographic, preoperative, oncological, and surgical characteristics of the population study divided into the two groups

\begin{tabular}{|c|c|c|c|}
\hline Variables & Patients $<70 n=50$ & Patients $>70 n=27$ & $p$ \\
\hline Median age & $62.5(35-69)$ & $73(70-79)$ & $<0.01$ \\
\hline Males & $29(58 \%)$ & $19(70.4 \%)$ & 0.33 \\
\hline ASA score & & & $<0.01$ \\
\hline 1 & $16(32 \%)$ & 0 & \\
\hline 2 & $12(24 \%)$ & $13(48.1 \%)$ & \\
\hline 3 & $22(44 \%)$ & $14(51.9 \%)$ & \\
\hline ECOG PS score & & & $<0.01$ \\
\hline 0 & $34(68 \% \%)$ & $10(37 \%)$ & \\
\hline 1 & $12(24 \%)$ & $16(59.3 \%)$ & \\
\hline 2 & $4(8 \%)$ & $1(3.7 \%)$ & \\
\hline CCI & & & 0.03 \\
\hline 0 & $21(42 \%)$ & $9(33.3 \%)$ & \\
\hline 1 & $7(14 \%)$ & $12(44.4 \%)$ & \\
\hline 2 & $13(26 \%)$ & $3(11.1 \%)$ & \\
\hline 3 & $8(16 \%)$ & $2(7.4 \%)$ & \\
\hline 4 & 0 & $1(3.7 \%)$ & \\
\hline 5 & $1(2 \%)$ & 0 & \\
\hline Mean FEV1\% & $82.96 \pm 18.39$ & $83.88 \pm 23.19$ & 0.88 \\
\hline Mean DLCO $\%$ & $66.55 \pm 15.43$ & $68 \pm 18.83$ & 0.79 \\
\hline Creatinine $\mathrm{mg} / \mathrm{dl}$ & $0.93 \pm 0.19$ & $0.9 \pm 0.21$ & 0.68 \\
\hline $\mathrm{Hb} \mathrm{gr} / \mathrm{dl}$ & $12.14 \pm 1.02$ & $11.85 \pm 2.16$ & 0.63 \\
\hline $\mathrm{RBC}$ & $3.91 \pm 0.45$ & $3.99 \pm 0.65$ & 0.67 \\
\hline WBC & $8015 \pm 2998$ & $8649 \pm 5307$ & 0.66 \\
\hline Number of comorbidities & & & 0.35 \\
\hline 0 & $14(28 \%)$ & $5(18.5 \%)$ & \\
\hline 1 & $13(26 \%)$ & $5(18.5 \%)$ & \\
\hline 2 & $10(20 \%)$ & $9(33.3 \%)$ & \\
\hline$>3$ & $13(26 \%)$ & $8(29.6 \%)$ & \\
\hline Comorbidities & & & 0.57 \\
\hline Hypertension & $11(25 \%)$ & $13(50 \%)$ & \\
\hline COPD & $15(34 \%)$ & $9(34.6 \%)$ & \\
\hline DM & $8(18 \%)$ & $3(11.5 \%)$ & \\
\hline Peripheral vascular disease & $3(6.8 \%)$ & $2(7.6 \%)$ & \\
\hline CAD & $2(4.5 \%)$ & $2(7.6 \%)$ & \\
\hline Other tumours & $3(6.8 \%)$ & $5(19.2 \%)$ & \\
\hline Pathology & & & 0.03 \\
\hline $\mathrm{ADC}$ & $31(62 \%)$ & $11(40.7 \%)$ & \\
\hline $\mathrm{SCC}$ & $12(24 \%)$ & $15(55.6 \%)$ & \\
\hline NSCLC NOS & $2(4 \%)$ & 0 & \\
\hline Other & $5(10 \%)$ & $1(3.7 \%)$ & \\
\hline Clinical stage & & & 0.72 \\
\hline $\mathrm{Ib}$ & $1(2 \%)$ & 0 & \\
\hline IIb & $3(6 \%)$ & $1(3.7 \%)$ & \\
\hline IIIa & $35(70 \%)$ & $22(81.5 \%)$ & \\
\hline IIIb & $10(20 \%)$ & $3(11.1 \%)$ & \\
\hline IV & $1(2 \%)$ & $1(3.7 \%)$ & \\
\hline $\mathrm{CHT}$ & $38(76 \%)$ & $26(96.3 \%)$ & 0.027 \\
\hline $\mathrm{CHT}+\mathrm{RT}$ & $12(24 \%)$ & $1(3.7 \%)$ & \\
\hline
\end{tabular}


Table 1 (continued)

\begin{tabular}{|c|c|c|c|}
\hline Variables & Patients $<70 n=50$ & Patients $>70 n=27$ & $p$ \\
\hline Type of chemotherapy & & & 0.24 \\
\hline Cddp & $40(80 \%)$ & $18(66.7 \%)$ & \\
\hline Cbdca & $7(14 \%)$ & $8(29.6 \%)$ & \\
\hline Other & $3(6 \%)$ & $1(3.7 \%)$ & \\
\hline Response to therapy & & & 0.48 \\
\hline Complete response & $6(12 \%)$ & $3(11.1 \%)$ & \\
\hline Partial response & $42(84 \%)$ & $21(77.8 \%)$ & \\
\hline Stable disease & $2(4 \%)$ & $3(11.1 \%)$ & \\
\hline Surgical procedure & & & 0.82 \\
\hline Pneumonectomy & $7(14 \%)$ & $5(18.5 \%)$ & \\
\hline Bilobectomy & $4(8 \%)$ & $1(3.7 \%)$ & \\
\hline Sleeve-lobectomy & $7(14 \%)$ & $5(18.5 \%)$ & \\
\hline Lobectomy & $28(56 \%)$ & $15(55.6 \%)$ & \\
\hline Segmentectomy & $2(4 \%)$ & $1(3.7 \%)$ & \\
\hline Wedge resection & $2(4 \%)$ & 0 & \\
\hline Side & & & 0.077 \\
\hline Right & $36(72 \%)$ & $14(51.9 \%)$ & \\
\hline Left & $14(28 \%)$ & $13(48.1 \%)$ & \\
\hline Side of pneumonectomy & & & 0.82 \\
\hline Right & $5(10 \%)$ & $4(14.8 \%)$ & \\
\hline Left & $2(4 \%)$ & $1(3.7 \%)$ & \\
\hline VATS approach & $8(16 \%)$ & $3(11.1 \%)$ & 0.73 \\
\hline Chest wall resection & $10(20 \%)$ & $3(11.1 \%)$ & 0.52 \\
\hline Pathological stage & & & 0.21 \\
\hline $\mathrm{CR}$ & $7(14 \%)$ & $3(11.1 \%)$ & \\
\hline Ia & $7(14 \%)$ & $3(11.1 \%)$ & \\
\hline $\mathrm{Ib}$ & $5(10 \%)$ & $2(7.4 \%)$ & \\
\hline IIa & $1(2 \%)$ & $3(11.1 \%)$ & \\
\hline IIb & $15(30 \%)$ & $5(18.5 \%)$ & \\
\hline IIIa & $15(30 \%)$ & $8(29.6 \%)$ & \\
\hline IIIb & 0 & $2(7.4 \%)$ & \\
\hline IVa & 0 & $1(3.7 \%)$ & \\
\hline
\end{tabular}

ASA American Society of Anesthesiologists, ECOG PS Eastern Cooperative Oncology Group Performance Status, CCI Charlson Comorbidity Index; FEV1\% Forced Expiratory Volume in $1 \mathrm{~s}, D L C O \%$ Diffusing Capacity of the Lung for Carbon Monoxide, $R B C$ red blood cells, $W B C$ white blood cells, $C O P D$ chronic obstructive pulmonary disease, $D M$ diabetes mellitus, $C A D$ coronary artery disease, $A D C$ adenocarcinoma, $S C C$ squamous cell carcinoma, NOS not otherwise specified, $C H T$ chemotherapy, $C H T+R T$ chemotherapy + radiotherapy, $C D D P$ cisplatin-based chemotherapy, $C B D C A$ carboplatin-based chemotherapy, VATS video-assisted thoracoscopic surgery, $C R$ complete response

The mortality rate ( $2 \%$ vs $0 \%$ ), the proportion of patients who suffered from any complication ( $38 \%$ vs $48.1 \%)$, median ICU stay ( 1 vs 1 day) and median length of stay (7 vs 8.5 days) were not statistically different between the two groups ( $p=0.45 ; p=0.47 ; p=0.5$ and 0.49 respectively). The overall complication rate was higher in group B $(50 \%$ vs $77 \%$ ) and in particular we observed a significantly higher incidence of atrial fibrillation ( $4 \%$ vs $25.9 \% ; p<0.01$ ). The grade of complications, measured with the Clavien-Dindo classification [23], was not different between the two groups (Table 2).
The ECOG PS score $\geq 1$ and chest wall resection were risk factors associated with complications at univariate analysis (OR $2.57 p=0.047$ and OR $4.011 p=0.034$ respectively). Despite the presence of some high OR values, the multivariable analysis demonstrated the absence of any significant factor associated with overall morbidity (Table 3 ). The minimally-invasive VATS approach showed a favourable association with complications in both univariate and multivariable analyses (OR 0.167, $p=0.023$ ).

At the median follow-up of 22 months (4-54 months), 52 (67.5\%) patients were alive and the OS at 3 years (Fig. 1) and 
Table 2 Post-operative outcomes

\begin{tabular}{llll}
\hline Characteristics & Patients $<70 n=50$ & Patients $>70 n=27$ & $p$ \\
\hline In-hospital mortality & $1(2 \%)$ & 0 & 0.45 \\
ICU stay (days), median and range & $1(1-30)$ & $1(1-160)$ & 0.5 \\
Length of stay (days), median and range & $7(3-35)$ & $8.5(4-180)$ & 0.49 \\
Patients with at least one complication & $19(38 \%)$ & $13(48.1 \%)$ & 0.47 \\
Complication rate & $25 / 50(50 \%)$ & $21 / 27(77 \%)$ & 0.01 \\
Grade of complications (Clavien-Dindo) & & & 0.6 \\
2 & $13(26 \%)$ & $9(33.3 \%)$ & \\
3a & $2(4 \%)$ & $2(7.4 \%)$ & \\
$3 b$ & 0 & 0 & \\
4a & $3(6 \%)$ & $1(3.7 \%)$ & \\
$4 b$ & 0 & $1(3.7 \%)$ & \\
5 & $1(2 \%)$ & 0 & 0.71 \\
Complications in detail & & & $<0.01$ \\
Respiratory failure & $7(14 \%)$ & $3(11.1 \%)$ & 0.5 \\
Atrial fibrillation & $2(4 \%)$ & $7(25.9 \%)$ & 0.29 \\
Prolonged air leak & $3(6 \%)$ & $1(3.7 \%)$ & 0.64 \\
Chylothorax & $2(4 \%)$ & 0 & 0.17 \\
Pneumonia and sputum retention & $4(8 \%)$ & $3(11.1 \%)$ & 0.29 \\
Bleeding requiring blood transfusion & $4(8 \%)$ & $5(18.5 \%)$ & 0.17 \\
Contralateral pneumothorax & $2(4 \%)$ & 0 & 0.17 \\
Renal failure requiring dialysis & 0 & $1(3.7 \%)$ & 0.45 \\
Broncho-pleural fistula & 0 & $1(3.7 \%)$ & \\
Subclavian vein thrombosis & $1(2 \%)$ & & \\
\hline
\end{tabular}

$I C U$ intensive care unit
DFS at 2 years (Fig. 2) were not different between the two groups (61\% vs $48.5 \%, p=0.64 ; 61.7 \%$ vs $44 \%, p=0.393$ ). The elderly group has a median OS and DFS of 22 months and 10 months, respectively, while in the Group A OS and DFS were 22.5 and 9.5 months. Excluding postoperative death $(n=1), 20$ patients $(83.3 \%)$ died for cancer relapse. We observed 24 (49\%) cancer recurrence in Group A and 14 $(51,9 \%)$ in Group B $(p=1)$ : distant metastasis developed in $14(58.3 \%)$ and $9(64.2 \%)$ patients, respectively.

\section{Discussion}

This retrospective study compared postoperative and midterm oncological outcomes of elderly patients, defined as patients aged $>70$ years, versus younger treated by a multimodality approach including surgery for LA-NSCLC. Our results demonstrated that IT in a selected cohort of elderly patients was not associated with increased postoperative mortality.

The overall complication rate was still higher for the elderly who often suffered from some post-operative complications, but non-life-threatening complications. The incidence of respiratory failure, pulmonary infections, or broncho-pleural fistula, the most frequent causes of disability, and associated with post-operative death, were low and similar between the two groups. Furthermore, the grade of overall complication, resumed with the Clavien-Dindo classification, was no different between the two groups, and also the majority of patients suffered from grade 2 complications (26\% vs 33\%) that we can consider as minor complications.

Although the univariate analysis demonstrated that chest wall resection and ECOG PS [21] higher than 1 were risk factors for overall morbidity, the multivariable analysis did not confirm these findings. Therefore, our outcomes clearly showed that older age per se is not a risk factor for morbidity neither on univariate and multivariable analysis.

To date, there are few published papers focused on surgical resection after IT in the geriatric population. Rivera et al. [1] conducted a match-analysis on data from a national database comparing the post-operative course of 81 elderly patients (defined as $>75$ years or older) with younger patients. Although the mortality was similar, the authors reported a significantly increased incidence of postoperative major complications $(22.2 \%$ vs $14.8 \%, p=0.03)$ and longer length of stay for elderly patients (14.9 vs 12 days, $p=<0.01$ ). The overall complication rate was $50 \%$ (minor complication $n=23$ + major complication $n=18$ ) absolutely comparable with our findings. According to the 

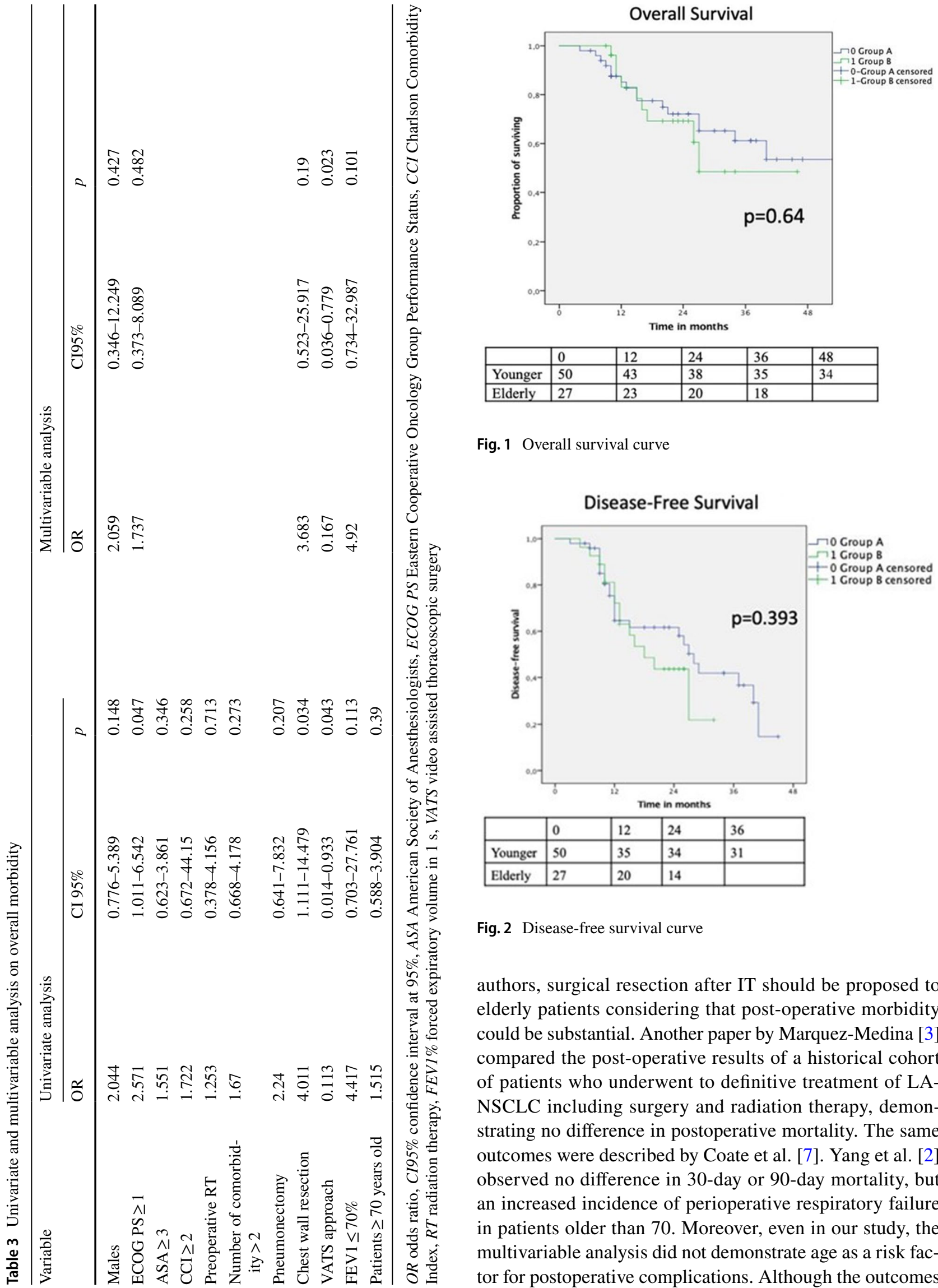

Fig. 1 Overall survival curve

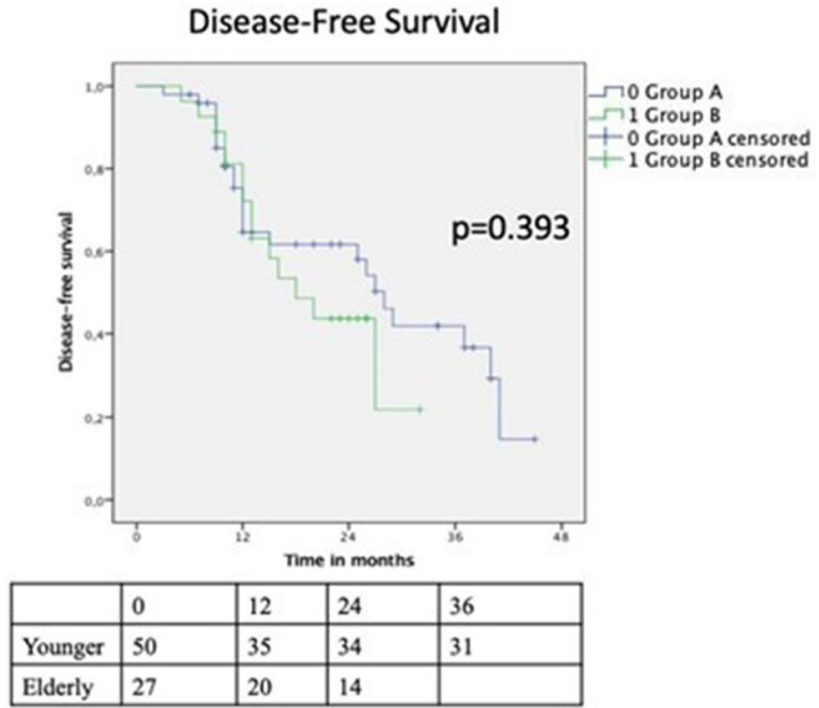

Fig. 2 Disease-free survival curve

authors, surgical resection after IT should be proposed to elderly patients considering that post-operative morbidity could be substantial. Another paper by Marquez-Medina [3] compared the post-operative results of a historical cohort of patients who underwent to definitive treatment of LANSCLC including surgery and radiation therapy, demonstrating no difference in postoperative mortality. The same outcomes were described by Coate et al. [7]. Yang et al. [2] observed no difference in 30-day or 90-day mortality, but an increased incidence of perioperative respiratory failure in patients older than 70. Moreover, even in our study, the multivariable analysis did not demonstrate age as a risk factor for postoperative complications. Although the outcomes 
of all these papers are encouraging, we must consider that all were retrospective historical studies with a long study period and also were biased by the relatively small population study, and lastly the definition of the elderly was heterogeneous between the studies.

The last evidence suggests that a bimodality approach (chemotherapy + radiation therapy VS chemotherapy + surgery) has not been shown to be superior to another in terms of overall survival in patients with clinical mediastinal $\mathrm{N} 2$ involvement [12, 13]. A non-significant advantage in disease-free survival was described in the Intergroup 0139 trial [15] in patients treated with the trimodality approach (chemotherapy + radiation therapy + surgery) compared with the bimodality treatment. Although in the cited randomized controlled trial elderly patients were not excluded, the geriatric population was underrepresented, and therefore few retrospective studies focused on the multimodality treatment in the elderly. Also in our population, radiation therapy was frequently administered to younger patients ( $24 \%$ vs $3.7 \%$, $p=0.027$ ) because this regimen is considered more aggressive and it could be not-well tolerated for elderly patients who often had fragile stability and some co-morbid conditions. Although not so much used in the whole study population, the preoperative radiation therapy did not have a strong impact on postoperative complications as showed at univariate analysis (Table 3).

Coate and coll [7] did not observe any overall statistically significant difference in survival between elderly and younger patients treated with curative intent. The same results were described by Yang [2] who also noted the absence of difference in the disease-free survival at univariate and multivariable analysis. Our series had a relatively short study period, but we had encouraging oncological results as shown by a median OS and DFS of 22 months and 10 months in both groups. Furthermore, the leading cause of death in both groups was recurrence and progression of cancer, not other comorbid conditions or diseases. These results demonstrate that age per se is not a contraindication for a curative-intent multimodality treatment and so this treatment could be proposed to a selected elderly population.

Our results and also data from the literature demonstrated that IT followed by major pulmonary resection for LANSCLC is a feasible approach in the elderly, but none had shown an algorithm to correctly select the patient to minimize the development of complications. The LA-NSCLC stage is a clinical scenario very heterogeneous and also the individual status of the elderly patient could be extremely variable and then only an individualized and tailored comprehensive evaluation could lead to the right selection. Several scores are available to assess the elderly status, but the personal and individual judgment is still basilar in this multidisciplinary setting.
Furthermore, it is very important to adopt a more surgical conservative attitude: first of all, avoiding pneumonectomy and being less invasive using for example the thoracoscopic approach. Some papers have demonstrated the oncological adequacy, safety, and effectiveness of the thoracoscopic approach even after IT [24, 25].

Our study has some limitations: (1) is a single institution series, retrospectively analyzed between a short period with a study population of patients who underwent only surgery as definitive treatment; (2) the patient cohort is heterogeneous for the presence of patients affected by superior sulcus tumor or by patients with chest wall involvement; (3) we did not perform a comparison with definitive radiation therapy; (4) lastly there is a lack of a structured follow-up investigation about daily activity level and quality of life. However, the short study period (2014-2018) could be interpreted as an advantage for the use of all the modern techniques of staging and re-staging (mostly the restaging with PET-CT and EBUS-EUS), for the adoption of modern surgical and anaesthetic approaches and also for the latest therapeutic protocols in both neoadjuvant and adjuvant settings.

\section{Conclusion}

In conclusion, the multimodality approach for LA-NSCLC could be applied to the geriatric population because age per se is not a risk factor for postoperative mortality and morbidity. Moreover, IT followed by surgery is a well-tolerated approach not associated with life-threatening postoperative complications, also in the elderly. More mid and long-term studies are necessary to investigate the oncological value of the multimodal approach in this population.

\section{Compliance with ethical standards}

Conflict of interest The authors declare that they do not have any conflict of interest.

Ethical approval This article does not contain any study with human participants or animals performed by any of the authors.

\section{References}

1. Rivera C, Jougon J, Dahan M, Falcoz PE, Bernard A, Brouchet L. Are postoperative consequences of neoadjuvant chemotherapy for non-small cell lung cancer more severe in elderly patients? Lung Cancer. 2012;76(2):216-21.

2. Yang CJ, Mayne NR, Wang H, et al. Outcomes of major lung resection after induction therapy for non-small cell lung cancer in elderly patients. Ann Thorac Surg. 2016;102(3):962-70. 
3. Marquez-Medina D, Martin-Marco A, Ojanguren-Garranz A. Age does not worsen the efficacy nor tolerance to combined induction therapies in locally advanced non-small cell lung cancer. Anticancer Res. 2014;34(8):4373-6.

4. Pallis AG, Gridelli C, Wedding U, Faivre-Finn C, Veronesi G, Jaklitsch M, Luciani A, O'Brien M. Management of elderly patients with NSCLC; updated expert's opinion paper: EORTC Elderly Task Force, Lung Cancer Group and International Society for Geriatric Oncology. Ann Oncol. 2014;25(7):1270-83.

5. Browner I, Purtell M. Chemotherapy in the older patient with operable non-small cell lung cancer: neoadjuvant and adjuvant regimens. Thorac Surg Clin. 2009;19(3):377-89.

6. Detillon DDEMA, Veen EJ. Postoperative outcome after pulmonary surgery for non-small cell lung cancer in elderly patients. Ann Thorac Surg. 2018;105(1):287-93.

7. Coate LE, Massey C, Hope A, Sacher A, Barrett K, Pierre A, Leighl N, Brade A, de Perrot M, Waddell T, Liu G, Feld R, Burkes R, Cho BC, Darling G, Sun A, Keshavjee S, Bezjak A, Shepherd FA. Treatment of the elderly when cure is the goal: the influence of age on treatment selection and efficacy for stage III non-small cell lung cancer. J Thorac Oncol. 2011;6(3):537-44.

8. Martins RG, D'Amico TA, Loo BW Jr, et al. The management of patients with stage IIIA non-small cell lung cancer with N2 mediastinal node involvement. J Natl Compr Canc Netw. 2012;10:599-613.

9. Shah AA, Berry MF, Tzao C, et al. Induction chemoradiation is not superior to induction chemotherapy alone in stage IIIA lung cancer. Ann Thorac Surg. 2012;93:1807-12.

10. Roth JA, Fossella F, Komaki R, et al. A randomized trial comparing perioperative chemotherapy and surgery with surgery alone in resectable stage IIIA non-small-cell lung cancer. J Natl Cancer Inst. 1994;86:673-80.

11. Scagliotti GV, Pastorino U, Vansteenkiste JF, et al. Randomized phase III study of surgery alone or surgery plus preoperative cisplatin and gemcitabine in stages IB to IIIA non-small-cell lung cancer. J Clin Oncol. 2012;30:172-8.

12. Song WA, Zhou NK, Wang W, et al. Survival benefit of neoadjuvant chemotherapy in non-small cell lung cancer: an updated meta-analysis of 13 randomized control trials. J Thorac Oncol. 2010;5:510-6.

13. Blumenthal GM, Bunn PA Jr, Chaft JE, et al. Current status and future perspectives on neoadjuvant therapy in lung cancer. $\mathrm{J}$ Thorac Oncol. 2018;13(12):1818-31.

14. Werner-Wasik M, Scott C, Cox JD, et al. Recursive partitioning analysis of 1999 Radiation Therapy Oncology Group (RTOG) patients with locally-advanced non-small-cell lung cancer (LANSCLC): identification of five groups with different survival. Int J Radiat Oncol Biol Phys. 2000;48(5):1475-82.

15. Albain KS, Swann RS, Rusch VW, et al. Radiotherapy plus chemotherapy with or without surgical resection for stage III non-smallcell lung cancer: a phase III randomised controlled trial. Lancet. 2009;374(9687):379-86.

16. Chansky K, Detterbeck FC, Nicholson AG, et al. The IASLC Lung Cancer Staging Project: external validation of the revision of the TNM stage groupings in the eighth edition of the TNM classification of lung cancer. J Thorac Oncol. 2017;12(7):1109-21.

17. National Comprehensive Cancer Network. Non-Small Cell Lung Cancer (Version 5.2018). https://www.nccn.org/

18. Postmus PE, Kerr KM, Oudkerk M, Senan S, Waller DA, Vansteenkiste J, Escriu C, Peters S. Early-stage and locally advanced (non-metastatic) non-small-cell lung cancer: ESMO clinical practice guidelines. Ann Oncol. 2017;28(suppl 4):1-21.

19. Hotta K, Ueoka H, Kiura K, Tabata M, Tanimoto M. An overview of 48 elderly-specific clinical trials of systemic chemotherapy for advanced non-small cell lung cancer. Lung Cancer. 2004;46(1):61-766.

20. Gridelli C, Langer C, Maione P, Rossi A, Schild SE. Lung cancer in the elderly. J Clin Oncol. 2007;25(14):1898-907.

21. Oken M, Creech R, Tormey D, et al. Toxicity and response criteria of the Eastern Cooperative Oncology Group. Am J Clin Oncol. 1982;5:649-55.

22. Asmis TR, Ding K, Seymour L, et al. Age and comorbidity as independent prognostic factors in the treatment of non small-cell lung cancer: a review of National Cancer Institute of Canada Clinical Trials Group trials. J Clin Oncol. 2008;26(1):54-9.

23. Dindo D, Demartines N, Clavien PA. Classification of surgical complications: a new proposal with evaluation in a cohort of 6336 patients and results of a survey. Ann Surg. 2004;240:205-13.

24. Gonfiotti A, Bongiolatti S, Bertolaccini L, et al. Thoracoscopic lobectomy for locally advanced-stage non-small cell lung cancer is a feasible and safe approach: analysis from multi-institutional national database. J Vis Surg. 2017;7(3):160.

25. Hennon M, Sahai RK, Yendamuri S, Tan W, Demmy TL, Nwogu C. Safety of thoracoscopic lobectomy in locally advanced lung cancer. Ann Surg Oncol. 2011;18(13):3732-6.

Publisher's Note Springer Nature remains neutral with regard to jurisdictional claims in published maps and institutional affiliations. 